\title{
Utilidad de la procalcitonina sérica para la discriminación temprana entre fascitis necrosante y celulitis de las extremidades: serie de casos y revisión de la literatura.
}

\author{
DOI: http://dx.doi.org/10.37315/SOTOCAV201928054128
}

SALAZAR J1, NOVOA-PARRA C 2; WADHWANI J1, MONTANER-ALONSO D1; RODRIGO-PÉREZ J1.

1 SERVICIO DE CIRUGÍA ORTOPÉDICA Y TRAUMATOLOGÍA. HOSPITAL UNIVERSITARIO DR. PESET. VALENCIA, ESPAÑA 2 SERVICIO DE CIRUGÍA ORTOPÉDICA Y TRAUMATOLOGÍA. HOSPITAL DE DENIA, ALICANTE, ESPAÑA

\begin{abstract}
Resumen.
Objetivo: valorar la utilidad de una escala de riesgo basada en la procalcitonina sérica al compararla con el LRINEC score en la discriminación temprana entre la fascitis necrosante (FN) y la celulitis de las extremidades.

Material y método: estudio retrospectivo, entre 2009 y 2017, de todos los pacientes con el diagnóstico confirmado de FN en una extremidad $(\mathrm{N}=10)$. Los hallazgos de estos pacientes se compararon con los de 23 pacientes que fueron ingresados por celulitis grave de miembros en el mismo periodo. Se analizaron las variables relacionadas con cada grupo. Mediante el área bajo la curva $(A B C)$, se comparó la capacidad de discriminación para el diagnóstico de FN de dos escalas, una basada en los niveles de procalcitonina y otra basada en la puntuación de la escala LRINEC.
\end{abstract}

Resultados: el área bajo la curva fue mayor para el riesgo categorizado por los niveles de procalcitonina. Al analizar el LRINEC score y los niveles de procalcitonina como variables continuas, esta última también presentó una mayor área bajo la curva. El punto de corte con mayor área bajo la curva fue el correspondiente a $>0,87 \mathrm{ng} / \mathrm{ml}$ de procalcitonina (sensibilidad del $100 \%$ y especificidad de $82,6 \%$ ) y la puntuación 5 en el LRINEC score (sensibilidad de $80 \%$ y especificidad de $82,6 \%$ ).

Conclusión: en nuestra muestra la medición del nivel de procalcitonina y la escala basada en este nivel se ha mostrado como una herramienta más sensible y por lo menos igual de específica que la escala LRINEC a la hora de discriminar de manera temprana entre una FN de una celulitis de las extremidades.

Palabras clave: Procalcitonina, Fascitis necrosante, Celulitis

\section{Summary.}

Objective: to assess the usefulness of a risk scale based on serum procalcitonin when compared with the LRINEC score in the early discrimination between necrotizing fasciitis (NF) and extremities cellulite.

Material and method: retrospective study, between 2009 and 2017, of all patients with the confirmed diagnosis of NF in one limb $(\mathrm{N}=10)$. The findings of these patients were compared with those of 23 patients who were admitted for severe cellulitis of limbs in the same period. The variables related to each group were analyzed. Using the area under the curve (AUC), the discrimination capacity for NF diagnosis of two scales was compared, one based on procalcitonin levels and another based on the LRINEC score.

Results: the area under the curve was greater for the risk categorized by procalcitonin levels. When analyzing the LRINEC score and procalcitonin levels as continuous variables, the latter also presented a larger area under the curve. The cut-off point with the greatest area under the curve was that corresponding to $>0.87 \mathrm{ng} / \mathrm{ml}$ of procalcitonin (sensitivity of $100 \%$ and specificity of $82.6 \%$ ) and score 5 on the LRINEC score (sensitivity of $80 \%$ and specificity of $82.6 \%$ ).

Conclusion: in our sample the level of procalcitonin and the scale based on this level has been shown as a more sensitive and at least as specific tool as the LRINEC scale for early discrimination between NF and a cellulite of the extremities.

\section{Correspondencia:}

Carlos Novoa Parra

Email: le_male2002@hotmail.com 
SALAZAR J Y COLS. Utilidad de la procalcitonina sérica para la discriminación temprana entre fascitis necrosante y celulitis de las extremidades: serie de casos y revisión de la literatura.

\section{Introducción}

La fascitis necrosante (FN) es una enfermedad mortal que justifica el diagnóstico inmediato y desbridamiento quirúrgico. Sin embargo, en ciertos casos es difícil diferenciar entre $\mathrm{FN}$ y celulitis ya que la presentación clínica de ambas entidades parece similar en la fase temprana. La escala Laboratory Risk Indicator for Necrotizing Fasciitis (LRINEC score) propuesta por Wong et al. en $2004^{1}$ ha sido validada en un intento de diagnosticar precozmente una $\mathrm{FN}$ al diferenciarla de otras infecciones de tejidos blandos. La puntuación total del LRINEC score varía de 0 a 13 , y el punto de corte de 6 puntos tuvo un valor predictivo positivo del $92 \%$ y un valor predictivo negativo del $96 \%{ }^{1}$.

La Procalcitonina (PCT) es un precursor peptídico de la hormona calcitonina, que está involucrada en la homeostasis del calcio. El nivel de PCT aumenta en respuesta a un estímulo proinflamatorio, especialmente de origen bacteriano ${ }^{2}$. Durante una infección bacteriana, la PCT se produce principalmente por las células de los pulmones y el intestino, y es un marcador confiable en el diagnóstico y tratamiento de infecciones bacterianas graves y sepsis ${ }^{3}$. Al-Thani et al [4] observaron una correlación positiva entre el LRINEC score y los niveles de procalcitonina en la predicción de shock séptico en pacientes con FN. Cabe destacar que ambos trabajos ${ }^{1,4}$ no fueron realizados únicamente en pacientes con afectación de las extremidades.

Nuestro objetivo es valorar la utilidad de una la escala de riesgo basada en la procalcitonina sérica al compararlo con el LRINEC score en la discriminación temprana entre la fascitis necrosante y la celulitis de las extremidades.

\section{Material y Métodos}

Se revisaron de manera retrospectiva las historias clínicas de todos los pacientes con el diagnóstico confirmado de FN en una extremidad entre 2009 y 2017 en nuestro centro. Se incluyeron en el estudio aquellos pacientes que, tras el diagnóstico clínico de sospecha de $\mathrm{FN}$ en el servicio de urgencias, presentaron durante el desbridamiento hallazgos quirúrgicos característicos de infección necrosante: fascia desvitalizada de fácil disección digital entre planos o finger test, presencia de exudado purulento o en "agua de lavar platos" (dishwater pus), ausencia de sangrado y trombosis vascular regional y tuvieron un diagnóstico histopatológico de fascitis necrosante. En el mismo periodo trescientos cincuenta y cinco pacientes ingresaron en nuestra institución con el diagnóstico clínico de celulitis o abscesos de las extremidades. Se revisaron la historias clínicas para identificar pacientes con infección grave de tejidos blandos: los criterios que utilizamos para las infecciones graves de tejidos blandos fueron la necesidad de hospitalización por más de 48 horas debido a la impresión clínica de infección severa, el uso de antibióticos parenterales durante $>48$ horas, la presencia de abscesos que necesitaron desbridamiento quirúrgico. Se excluyeron los pacientes con una hospitalización $<48$ horas y el uso solo de antibióticos orales, ya que se consideró que estos pacientes tenían infecciones menores de tejidos blandos. Cincuenta y cinco pacientes fueron seleccionados. De estos, en 23 pacientes se solicitó la procalcitonina sérica en el momento del ingreso y se utilizaron como controles para este estudio.

Se recogieron los siguientes datos: sexo, edad, extremidad afectada, comorbilidades, parámetros para calcular el LRINEC (PCR, creatinina, hemoglobina, recuento de leucocitos, glucosa, sodio sérico) y los niveles de procalcitonina al ingreso. Además se registraron el número de amputaciones y mortalidad. El riesgo de padecer $\mathrm{FN}$ de los pacientes lo categorizamos según el LRINEC [1] en bajo riesgo menor de 6, moderado riesgo: 6-7 y alto riesgo: mayor de 7 , y según los niveles de procalcitonina en bajo riesgo $<0,5 \mathrm{ng} / \mathrm{mL}$, moderado riesgo $\geq 0,5-<2 \mathrm{ng} / \mathrm{mL}$, alto riesgo $\geq 2-<10$ $\mathrm{ng} / \mathrm{mL}$ y muy alto riesgo $\geq 10 \mathrm{ng} / \mathrm{mL}$ [4].

Los resultados se muestran como mediana y rango intercuartílico para las variables cuantitativas. En el caso de las variables cualitativas, los resultados se muestran como frecuencia absoluta. La $U$ de MannWhitney se utilizó para las comparaciones entre variables cuantitativas. La prueba exacta de Fisher y la razón de verosimilitud se utilizaron para la comparación de las variables cualitativas. Se utilizó la correlación de Sperman para analizar la relación entre las categorías de riesgo. Se utilizó el área bajo la curva $(A B C)$ para determinar la capacidad de discriminación entre FN y celulitis de las escalas basadas en el LRINEC score y en los niveles de PCT. Los puntos de corte con mayor $A B C$ se determinaron mediante el índice de Youden. El Análisis estadístico se llevó a cabo mediante el uso del programa Statistical Package for the Social Sciences (SPSS $尺)$ V.22.

\section{Resultados}

Los grupos fueron comparables en cuanto a sus características personales. Encontramos diferencias estadísticamente significativas entre los grupos en el nivel de hemoglobina, el LRINEC score y el nivel de procalcitonina. Encontramos diferencias estadísticamente significativas entre los grupos en el riesgo categorizado mediante LRINEC score $y$ procalcitonina, siendo mayor el riesgo en los casos en los que el diagnóstico fue FN. En toda la muestra se presentaron 3 muertes y 3 amputaciones, todas en el grupo de FN ( $p=0,022)$. El grupo de pacientes con FN tuvo un mayor tiempo de hospitalización con una mediana de 24 días en comparación con los 3 días del grupo de celulitis. Estos resultados se encuentran resumidos en la Tabla I.

El riesgo categorizado mediante LRINEC score y mediante los niveles de procalcitonina tuvieron una relación directa moderada, correlación de Spearman de $0,55, \quad \mathrm{p} \quad 0,001$. 
SALAZAR J Y COLS. Utilidad de la procalcitonina sérica para la discriminación temprana entre fascitis necrosante y celulitis de las extremidades: serie de casos y revisión de la literatura.

Tabla I: comparaciones entre los grupos de pacientes con diagnóstico de fascitis necrosante y celulitis de las extremidades. *Estadísticamente significativo

\begin{tabular}{|c|c|c|c|}
\hline Variable & $\mathrm{FN}[\mathrm{N}=10]$ & Celulitis [N=23] & $p$ \\
\hline Sexo (mujer/hombre) & $5 / 5$ & $9 / 14$ & 0,707 \\
\hline Edad. Mediana (RIQ) & $51(44)$ & $60(29,7)$ & 0,475 \\
\hline Extremidad (Inferior/Superior) & $7 / 3$ & $18 / 5$ & 0,673 \\
\hline $\mathrm{DM}(\mathrm{Si} / \mathrm{No})$ & $3 / 7$ & $7 / 16$ & 0,999 \\
\hline VIH (Si/No) & $1 / 9$ & $1 / 22$ & 0,521 \\
\hline $\mathrm{VHC}(\mathrm{Si} / \mathrm{No})$ & $2 / 8$ & $1 / 22$ & 0,212 \\
\hline ICC (Si/No) & $2 / 8$ & $3 / 20$ & 0,627 \\
\hline IRC $(\mathrm{Si} / \mathrm{No})$ & $2 / 8$ & $1 / 22$ & 0,212 \\
\hline Cirrosis hepática (Si/No) & $1 / 9$ & $0 / 23$ & 0,303 \\
\hline $\mathrm{AR}(\mathrm{Si} / \mathrm{No})$ & $0 / 10$ & $1 / 22$ & 0,999 \\
\hline PCR (mg/L). Mediana (RIQ) & $201(194,5)$ & $122(127,6)$ & 0,057 \\
\hline Creatinina (mg/dL). Mediana (RIQ) & $1,48(1,72)$ & $0,86(0,19)$ & $0,038^{*}$ \\
\hline Hemoglobina (gr/dL). Mediana (RIQ) & $11,85(3,70)$ & $13,8(2,50)$ & $0,034 *$ \\
\hline Recuento de leucocitos (X10e9/L). Mediana (RIQ) & $14,5(7,9)$ & $11,9(7,8)$ & 0,802 \\
\hline Glucosa (mg/dL). Mediana (RIQ) & $124,5(81,5)$ & $119(49)$ & 0,923 \\
\hline Sodio sérico (meq/L). Mediana (RIQ) & $133,5(11)$ & $138(4)$ & 0,167 \\
\hline LRINEC. Mediana (RIQ) & $6(2)$ & $2(3)$ & $0,001 *$ \\
\hline Riesgo LRINEC Score (Bajo/ Moderado/Alto) & $2 / 6 / 2$ & $19 / 3 / 1$ & $0,002 *$ \\
\hline Procalcitonina (ng/ml). Mediana (RIQ) & $12,15(64,82)$ & $0,22(0,45)$ & $<0,001^{*}$ \\
\hline Riesgo Procalcitonina (Bajo/Moderado/Alto/Muy Alto) & $0 / 2 / 3 / 5$ & $15 / 5 / 2 / 1$ & $<0,001 *$ \\
\hline Mortalidad (Si/No) & $3 / 7$ & $0 / 23$ & $0,022 *$ \\
\hline Amputaciones (Si/No) & $3 / 7$ & $0 / 23$ & $0,022 *$ \\
\hline Días totales de ingreso hospitalario. Mediana (RIQ) & $23,5(37)$ & $3(3)$ & $<0,001^{*}$ \\
\hline
\end{tabular}


SALAZAR J Y COLS. Utilidad de la procalcitonina sérica para la discriminación temprana entre fascitis necrosante y celulitis de las extremidades: serie de casos y revisión de la literatura.

Tabla II: Variables descritas por estudios previos que estudiaron solo infecciones necrosantes de las extremidades. TP (tiempo de protrombina). ALERTS (Abnormal Liver function, Extent of infection, Renal impairment, Thrombocytopenia, and Shock). PCT (procalcitonina). Estudios donde se incluyó como variable el LRINEC score $\left({ }^{\star}\right)$ o PCT $\left({ }^{* *}\right)$

\begin{tabular}{|c|c|c|c|c|c|}
\hline Estudio & País & Año & $\begin{array}{l}\text { infecciones } \\
\text { necrosantes }\end{array}$ & Diagnóstico de FN & $\begin{array}{l}\text { Pronostico de: } \quad \text { Mortalidad }(\mathrm{M}) \text {; } \\
\text { Amputación (A); Estancia hospitalaria }(\mathrm{E})\end{array}$ \\
\hline $\begin{array}{l}\text { Tang et al } \\
{[12]}\end{array}$ & $\begin{array}{l}\text { Hong } \\
\text { Kong }\end{array}$ & 2001 & 24 & & $\begin{array}{l}\text { (M) Localización en la parte proximal del } \\
\text { miembro }\end{array}$ \\
\hline Tsai et al [13] & Taiwán & 2004 & 13 & & $\begin{array}{l}\text { (M) Presión arterial sistólica menor de } 90 \\
\text { mmHg, Contaje de glóbulos blancos } \\
\text { (disminución) }\end{array}$ \\
\hline $\begin{array}{l}\text { Wang y Hung } \\
{[10]}\end{array}$ & Taiwán & 2004 & 19 & Saturación de oxigeno $<70 \%$ & \\
\hline $\begin{array}{l}\text { Zahar et al } \\
{[11]}\end{array}$ & Francia & 2004 & 21 & $\begin{array}{l}\text { Shock, cianosis, necrosis } \\
\text { cutánea y presencia de } \\
\text { vesículas cutáneas. }\end{array}$ & $\begin{array}{l}\text { (M) La necesidad de ventilación mecánica y } \\
\text { diálisis. }\end{array}$ \\
\hline $\begin{array}{l}\text { Cheng et al } \\
{[14]}\end{array}$ & Taiwán & 2005 & 17 & & $\begin{array}{l}\text { (M) DM y el grado C de Child (enfermedad } \\
\text { hepática). }\end{array}$ \\
\hline $\begin{array}{l}\text { Ozalay et al } \\
{[15]}\end{array}$ & Turquía & 2006 & 22 & & (M) Edad \\
\hline Tsai et al [16] & Taiwán & 2007 & 32 & & $\begin{array}{l}\text { (M) Albumina sérica baja, la combinación } \\
\text { de enfermedad hepática y DM }\end{array}$ \\
\hline $\begin{array}{l}\text { Cheng et al } \\
{[17]}\end{array}$ & Taiwán & 2008 & 14 & & $\begin{array}{l}\text { (M) Disnea y alteración del estado de } \\
\text { conciencia al ingreso. }\end{array}$ \\
\hline $\begin{array}{l}\text { Hankins y } \\
\text { Souther [18] }\end{array}$ & USA & 2008 & 31 & & $\begin{array}{l}\text { (M) El grado de extensión de la necrosis al } \\
\text { ingreso }\end{array}$ \\
\hline $\begin{array}{l}\text { Chee et al } \\
{[19]}\end{array}$ & Malasia & 2009 & 41 & & (M) Edad y los niveles altos de creatinina \\
\hline $\begin{array}{l}\text { Khanna et al } \\
{[20]}\end{array}$ & India & 2009 & 118 & & $\begin{array}{l}\text { (M) Crepito (gas), shock, síndrome de } \\
\text { disfunción orgánica, embolismo pulmonar }\end{array}$ \\
\hline $\begin{array}{l}\text { Yeung et al } \\
{[21]^{*}}\end{array}$ & $\begin{array}{l}\text { Hong } \\
\text { Kong }\end{array}$ & 2011 & 29 & & $\begin{array}{l}\text { (M) Shock, la afectación en el codo o por } \\
\text { encima, recuento de plaquetas }<150 \text {, } \\
\text { creatinina }>1.5 \mathrm{mg} / \mathrm{dl} \text {, función hepática } \\
\text { alterada. ALERTS }>3\end{array}$ \\
\hline Tsai et al [22] & Taiwán & 2011 & 115 & & $\begin{array}{l}\text { (M) La infección por Vibrio } \\
\text { Vulnificus progresa más rápido y es más } \\
\text { letal que la infección por Staphylococcus } \\
\text { aureus }\end{array}$ \\
\hline $\begin{array}{l}\text { Espadar et al } \\
\text { [23] }\end{array}$ & Irán & 2011 & 24 & & $\begin{array}{l}\text { (M) Hiponatremia, hiperkalemia y aumento } \\
\text { de bandas en las series leucitarías / (A)DM } \\
\text { (mayor número de amputaciones) Infección } \\
\text { por gram+ (menor número de } \\
\text { amputaciones) }\end{array}$ \\
\hline
\end{tabular}


SALAZAR J Y COLS. Utilidad de la procalcitonina sérica para la discriminación temprana entre fascitis necrosante y celulitis de las extremidades: serie de casos y revisión de la literatura.

\begin{tabular}{|c|c|c|c|c|c|}
\hline $\begin{array}{l}\text { Chen et al } \\
{[24]}\end{array}$ & Taiwán & 2015 & 110 & & $\begin{array}{l}\text { (A) Amputación menor= la PCR y los } \\
\text { estados de Wegner 4-5. Amputación } \\
\text { mayor= los niveles de albumina y los } \\
\text { estados de Wegner 4-5. }\end{array}$ \\
\hline Lee et al[25] & Taiwán & 2016 & 18 & & $\begin{array}{l}\text { (M) Afectación de ambos miembros } \\
\text { inferiores, contaje leucocitario }<12.000 \\
10^{3} / \mu \mathrm{L} \text { y la trombocitopenia. }\end{array}$ \\
\hline $\begin{array}{l}\text { Corona et al } \\
{[26]^{*}}\end{array}$ & España & 2016 & 20 & & $\begin{array}{l}\text { (M) Creatinina (elevación) y la glucosa } \\
\text { sérica (disminución) }\end{array}$ \\
\hline $\begin{array}{l}\text { Kato et al } \\
{[9]^{*}, * *}\end{array}$ & Japón & 2016 & 3 & $\begin{array}{l}\text { El nivel de PCT fue más alto } \\
\text { en los pacientes con Infección } \\
\text { necrosante que en aquellos } \\
\text { con celulitis y estuvo } \\
\text { relacionado con el desarrollo } \\
\text { de shock séptico. }\end{array}$ & \\
\hline $\begin{array}{l}\text { Ballesteros et } \\
\text { al [7]* }\end{array}$ & España & 2017 & 24 & & $\begin{array}{l}\text { (E) El nivel alto en la escala LRINEC } \\
\text { triplica el número de días de hospitalización } \\
\text { con respecto al nivel bajo. }\end{array}$ \\
\hline
\end{tabular}

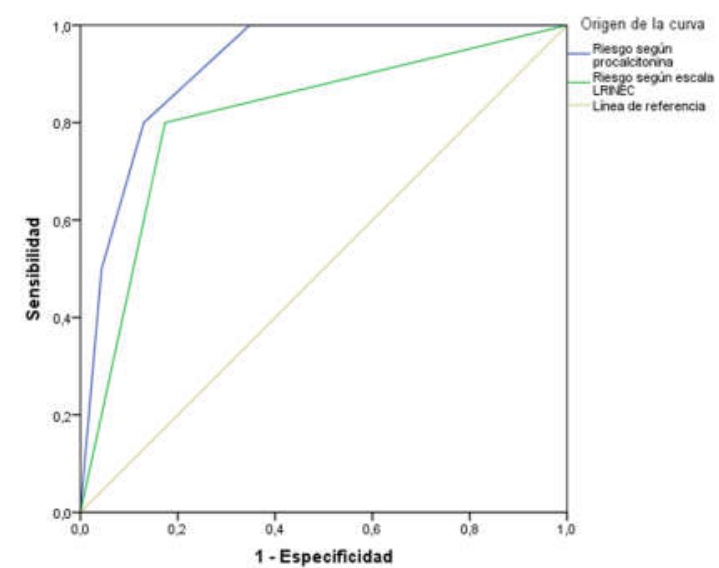

Figura 1: Curva COR de la capacidad de discriminación en el diagnóstico de FN según el riesgo calculado por LRINEC score (ABC 0,81; IC95\% 0,64-0,98) vs el riesgo calculado por el nivel de procalcitonina $(A B C 0,91$; IC95\% 0,82-1) como variables categóricas.

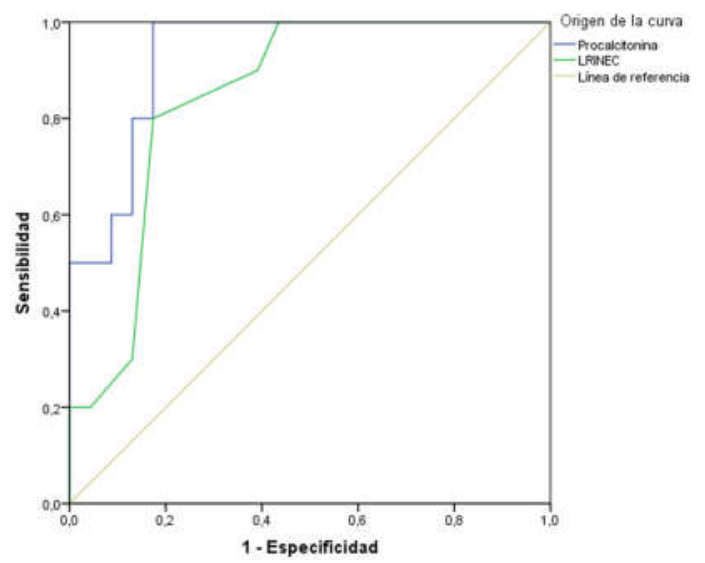

Figura 2: Curva COR de la capacidad de discriminación en el diagnóstico de FN según la puntuación LRINEC score (ABC 0,84; IC95\% 0,71-0,97) vs el nivel de procalcitonina (ABC 0,93; IC95\% 0,84-1) como variables continuas. 
SALAZAR J Y COLS. Utilidad de la procalcitonina sérica para la discriminación temprana entre fascitis necrosante y celulitis de las extremidades: serie de casos y revisión de la literatura.

El área bajo la curva fue mayor para el riesgo categorizado por los niveles de procalcitonina (Fig. 1). Al analizar el LRINEC score y los niveles de procalcitonina como variables continuas, esta última también presentó una mayor área bajo la curva. El punto de corte con mayor área bajo la curva fue el correspondiente a $>0,87 \mathrm{ng} / \mathrm{ml}$ de procalcitonina (sensibilidad del $100 \%$ y especificidad de $82,6 \%$ ) y la puntuación 5 en el LRINEC score (sensibilidad de $80 \%$ y especificidad de 82,6\%). (Fig. 2).

\section{Discusión}

Después que Wong et al ${ }^{1}$ propusieran el LRINEC score como una herramienta capaz de ayudar al diagnóstico temprano de $\mathrm{FN}$, ha habido estudios que han informado de su utilidad en diagnóstico temprano de la misma ${ }^{5}$. Su et $\mathrm{al}^{6}{ }^{6}$, no fueron capaces de encontrar relación entre el LRINEC y el diagnostico de FN, aunque si informaron de su utilidad en el pronóstico, donde una puntuación mayor a 6 se encontraba relacionada con una mayor mortalidad y numero de amputaciones. Es importante hacer relevante que de los estudios que conocemos en los que solo se hayan analizado $\mathrm{FN}$ de las extremidades, el LRINEC score solo se ha mostrado como una herramienta en la que sus valores altos se encuentran asociados a una mayor estancia hospitalaria 7. (Tab. II). Esta disparidad de resultados ha hecho que se analicen otros posibles marcadores, como variables asociadas o predictores, para el diagnóstico temprano de la FN. Al-Thani et al ${ }^{4}$ observaron que la procalcitonina puede servir como un marcador pronóstico para el desarrollo de shock séptico en pacientes con FN. Friederichs et al $^{8}$, observaron que podía ser utilizada como un marcador de éxito del primer tratamiento quirúrgico en la erradicación de la infección. En nuestro estudio hemos observado que la medición de los niveles de procalcitonina y la categorización del riego mediante la escala utilizada por Al-Thani et al ${ }^{4}$, es mejor herramienta que la escala LRINEC al momento de discriminar entre una $F N$ vs celulitis de las extremidades.

Hemos encontrado una correlación moderada entre la escala LRINEC y la escala de riesgo basada en la procalcitonina, algo curioso al pensar que ambas están diseñadas para analizar el riesgo de padecer infecciones graves y solo se han correlacionado en la mitad de los casos. La escala LRINEC parte con la ventaja de estar validada por un estudio bien diseñado en el cual se analizaron 8 veces más pacientes que en el nuestro, aunque como hemos destacado previamente no se ha podido corroborar en otras poblaciones y no solo analiza afectación de las extremidades.
Kato et al ${ }^{9}$, encontraron que el nivel de PCT fue más alto en los pacientes con una infección necrosante que en aquellos con celulitis de las extremidades. Al igual que en este estudio (10 pacientes), su muestra es pequeña (3 pacientes), aunque para nuestro conocimiento es el único trabajo previo al nuestro que considera la PCT como un marcador diagnostico en la FN de las extremidades. Otras variables asociadas al diagnóstico de $\mathrm{FN}$ en las extremidades, fueron encontradas por Wang y Hung ${ }^{10}$ y Zahar et $\mathrm{al}^{11}$. Los primeros encontraron asociación entre una saturación de oxigeno $<70 \%$ y el diagnostico de $\mathrm{FN}$, los segundos encontraron una asociación entre variables clínicas y el diagnostico de FN. (Tabla II). Lo cual nos lleva a pensar que en el diagnóstico de esta entidad tanto las variables clínicas como analíticas son igual de importantes y complementarias.

Las limitaciones de nuestro estudio en primer lugar está el bajo número de pacientes, aunque al ser esta entidad poco prevalente es difícil tener un tamaño muestral mayor. Al valorar los estudios previos, el promedio de pacientes incluidos es de 36 (rango 3-118) pacientes por estudio. En los estudios realizados en Europa el promedio es de 22 (rango 20-24) pacientes por estudio, con lo cual nuestra muestra no dista de manera extrema de las previas; en segundo lugar el ser un estudio observacional de cohorte retrospectiva; en tercer lugar el punto de corte de la procalcitonina valorada como una variable continua es bajo, y al tratarse de infecciones graves, sugerimos debe ser valorado en el contexto de los signos clínicos descritos por Wang y Hung ${ }^{10}$ y Zahar et al ${ }^{11}$. No obstante, consideramos que en la situación actual, en la que no disponemos de una herramienta que facilite de manera efectiva la identificación de una $\mathrm{FN}$, ante una infección de piel y partes blandas, como es la celulitis, la escala de riesgo basada en la medición de los niveles de procalcitonina nos puede ayudar, junto con los signos clínicos, a indicar un tratamiento precoz y agresivo.

\section{Conclusiones}

En conclusión, en nuestra muestra la medición del nivel de procalcitonina y la escala basada en este nivel se ha mostrado como una herramienta más sensible y por lo menos igual de específica que la escala LRINEC a la hora de discriminar de manera temprana entre una FN de una celulitis de las extremidades. 
SALAZAR J Y COLS. Utilidad de la procalcitonina sérica para la discriminación temprana entre fascitis necrosante y celulitis de las extremidades: serie de casos y revisión de la literatura.

\section{Bibliografía}

1. Wong $\mathrm{CH}$, Khin LW, Heng KS, Tan KC, Low CO. The LRINEC (Laboratory Risk Indicator for Necrotizing Fasciitis) score: a tool for distinguishing necrotizing fasciitis from other soft tissue infections. Crit Care Med 2004; 32:1535-41.

2. Linscheid P, Seboek D, Nylén ES, Langer I, Schlatter M, Becker KL, et al. In vitro and in vivo calcitonin I gene expression in parenchymal cells: a novel product of human adipose tissue. Endocrinology 2003;144:5578-84.

3. Mehanic S, Baljic R. The importance of serum procalcitonin in diagnosis and treatment of serious bacterial infections and sepsis. Mater Sociomed 2013; 25:277-81.

4. Al-Thani H, El-Menyar A, Shaikh N, Mudali I, Mekkodathil A, Asim M et al. Risk stratification of necrotizing fasciitis based on the initial procalcitonin concentration: a single center observational study. Surg Infect (Larchmt) 2015; 16(6):806-12.

5. Bechar J, Sepehripour S, Hardwicke J, Filobbos G. Laboratory risk indicator for necrotising fasciitis (LRINEC) score for the assessment of early necrotising fasciitis: a systematic review of the literature. Ann R Coll Surg Engl 2017; 99(5):341-6.

6. Su $\mathrm{Y}$, Chen $\mathrm{H}$, Hong $\mathrm{Y}$, Chen $\mathrm{C}$, Hsiao $\mathrm{C}$, Chen I. Laboratory risk indicator for necrotizing fasciitis score and the outcomes. ANZ J Surg 2008; 78(11):968-72.

7. Ballesteros-Betancourt J, García-Tarriño R, Ríos-Guillermo J, Rodriguez-Roiz J, Camacho P, Zumbado-Dijeres A et al. Infecciones necrosantes de partes blandas atendidas en un servicio de urgencias de tercer nivel: evolución y correlación con la escala laboratory risk indicator for necrotizing fasciitis (LRINEC). Rev Esp Cir Ortop Traumatol 2017; 61(4):265-72.

8. Friederichs J, Hutter M, Hierholzer C, Novotny A, Friess H, Bühren V et al. Procalcitonin ratio as a predictor of successful surgical treatment of severe necrotizing soft tissue infections. Am J Surg 2013; 206(3):368-73.

9. Kato T, Fujimoto N, Honda S, Fujii N, Shirai M, Nakanishi T et al. Usefulness of serum procalcitonin for early discrimination between necrotizing fasciitis and cellulitis. Acta Derm Venereol 2017; 97(1):141-2.

10. Wang TL, Hung CR. Role of tissue oxygen saturation monitoring in diagnosing necrotizing fasciitis of the lower limbs. Ann Emerg Med 2004; 44(3):222-8.

11. Zahar JR, Goveia J, Lesprit P, Brun-Buisson C. Severe soft tissue infections of the extremities in patients admitted to an intensive care unit. Clin Microbiol Infect 2005; 11(1):79-82.

12. Tang WM, Ho PL, Fung KK, Yuen KY, Leong JC. Necrotising fasciitis of a limb. J Bone Joint Surg Br 2001; 83(5):709-14.

13. Tsai YH, Hsu RW, Huang KC, Chen CH, Cheng CC, Peng KT, Huang TJ. Systemic Vibrio infection presenting as necrotizing fasciitis and sepsis. A series of thirteen cases. J Bone Joint Surg Am 2004; 86-A(11):2497-502.

14. Cheng NC, Tai HC, Tang YB, Chang SC, Wang JT. Necrotising fasciitis: clinical features in patients with liver cirrhosis. Br J Plast Surg 2005; 58(5):702-7.

15. Ozalay M, Ozkoc G, Akpinar S, Hersekli MA, Tandogan RN. Necrotizing soft-tissue infection of a limb: clinical presentation and factors related to mortality. Foot Ankle Int 2006; 27(8):598-605.

16. Tsai YH, Hsu RW, Huang TJ, Hsu WH, Huang KC, Li YY, Peng KT. Necrotizing soft-tissue infections and sepsis caused by Vibrio vulnificus compared with those caused by Aeromonas species. J Bone Joint Surg Am 2007; 89(3):631-6.

17. Cheng NC, Su YM, Kuo YS, Tai HC, Tang YB. Factors affecting the mortality of necrotizing fasciitis involving the upper extremities. Surg Today 2008; 38(12):1108-13.

18. Hankins $\mathrm{CL}$, Southern $\mathrm{S}$. Factors that affect the clinical course of group A beta-haemolytic streptococcal infections of the hand and upper extremity: a retrospective study. Scand J Plast Reconstr Surg Hand Surg 2008; 42(3):153-7.

19. Chee E, Kwan M, Khoo E. Necrotizing Fascitis of the Lower Limb - A Prospective Study of Prognostic Factors Affecting Mortality. Malaysian Orthopaedic Journal 2009; 3(1):32-5.

20. Khanna AK, Tiwary SK, Kumar P, Khanna R, Khanna A. A case series describing 118 patients with lower limb necrotizing fasciitis. Int J Low Extrem Wounds 2009; 8(2):112-6.

21. Yeung YK, Ho ST, Yen CH, Ho PC, Tse WL, Lau YK, et al. Factors affecting mortality in Hong Kong patients with upper limb necrotising fasciitis. Hong Kong Med J 2011; 17(2):96-104. 
SALAZAR J Y COLS. Utilidad de la procalcitonina sérica para la discriminación temprana entre fascitis necrosante y celulitis de las extremidades: serie de casos y revisión de la literatura.

22. Tsai YH, Wen-Wei Hsu R, Huang KC, Huang TJ. Comparison of necrotizing fasciitis and sepsis caused by Vibrio vulnificus and Staphylococcus aureus. J Bone Joint Surg Am 2011; 93(3):274-84.

23. Espandar R, Sibdari S, Rafiee E, Yazdanian S. Necrotizing fasciitis of the extremities: a prospective study. Strategies Trauma Limb Reconstr 2011; 6(3):121-25.

24. Chen IW, Yang HM, Chiu CH, Yeh JT, Huang CH, Huang YY. Clinical Characteristics and Risk Factor Analysis for Lower-Extremity Amputations in Diabetic Patients With Foot Ulcer Complicated by Necrotizing Fasciitis. Medicine (Baltimore) 2015; 94(44):e1957.

25. Lee CY, Li YY, Huang TW, Huang TY, Hsu WH, Tsai YH, Huang JC, Huang KC. Synchronous multifocal necrotizing fasciitis prognostic factors: a retrospective case series study in a single center. Infection 2016; 44(6):757-63.

26. Corona PS, Erimeiku F, Reverté-Vinaixa MM, Soldado F, Amat C, Carrera L. Necrotising fasciitis of the extremities: implementation of new management technologies. Injury 2016; 47 Suppl 3:S66-S71. 